\title{
A plugin-based approach to data analysis for the AMS ex- periment on the ISS
}

\author{
Valerio Formato $^{1, *}$ \\ ${ }^{1}$ INFN - Sezione di Perugia, Via A. Pascoli s.n.c., 06123 Perugia (PG)
}

\begin{abstract}
In many HEP experiments a typical data analysis workflow requires each user to read the experiment data in order to extract meaningful information and produce relevant plots for the considered analysis. Multiple users accessing the same data result in a redundant access to the data itself, which could be factorized effectively improving the CPU efficiency of the analysis jobs and relieving stress from the storage infrastructure. To address this issue we present a modular and lightweight solution where the users code is embedded in different "analysis plugins" which are then collected and loaded at runtime for execution, where the data is read only once and shared between all the different plugins. This solution was developed for one of the data analysis groups within the AMS collaboration but is easily extendable to all kinds of analyses and workloads that need $\mathrm{I} / \mathrm{O}$ access on AMS data or custom data formats and can even adapted with little effort to another HEP experiment data. This framework could then be easily embedded into a "analysis train" and we will discuss a possible implementation and different ways to optimise CPU efficiency and execution time.
\end{abstract}

\section{Introduction}

In a typical High-Energy physics (HEP) experiment the analysis of the collected data is often organised into different groups of people who collaborate towards a single measurement of a particular physics topic. This workflow usually results in people tackling different problems of the same analysis concurrently, in order to efficiently split the workload and to obtain a meaningful physics result quicker. Also, to avoid biases and to ensure the reproducibility of the result, people in the same analysis group share the same exact dataset to work on.

A common realization of this kind of workflow is the one where the people involved in the analysis will all read from the same dataset, perform some operations on the data they read, and write out the result of such operations on some new files. The underlying issue with this approach is in the fact that the data access is completely replicated between people in a redundant manner. Combined with the fact that in HEP analysis processes I/O access to data on disk constitutes a significant fraction of the wall-clock time, this usually results in a inefficient use of the computing resources allocated for the analysis.

In this work an analysis framework developed for the data analysis of the AMS-02 [1] collaboration is presented, that allows people involved in the analysis to share the data-access at runtime.

\footnotetext{
*e-mail: valerio.formato@cern.ch
} 


\section{The framework}

In our framework a clear separation is made between the process of loading the AMS data from file and the execution of user code on the data that has been loaded. The user code is encapsulated in a "plugin" which is dynamically loaded by the main executable which, in turn, has the only task of reading the event data and feeding it to the various plugins.

The framework is heavily based on the R00T [2] TSelector class: in the main executable a manager class, derived from TSelector, handles the registration and execution of all the user plugins (also derived from TSelector).

The choice of the TSelector class was adopted because it enforces a modular approach to the analysis, and maps very well to the most common steps in HEP data analysis (see Figure 1).

\begin{tabular}{|l|}
\hline TSelector: : Begin() \\
TSelector: : SlaveBegin( )
\end{tabular}

Figure 1. Parallelism between the common steps in HEP data analysis (left) and the TSelector structure (right).

\subsection{The plugin manager}

The core of the framework is the TSAnaPluginManager class (hereafter also called "plugin manager"), a custom class derived from TSelector. It serves mainly two functions: it loads and stores in memory the data to be processed, and it serves the data to the user plugins it has registered for execution.

In this particular case study the data is stored in standard ROOT files using a standard TTree where each TBranch holds a data container each specialised to hold data from a particular subdetector. The TSAnaPluginManager also inherits from a custom Event class, which internally holds pointers to all the data structures in the tree branches. The idea behind this double inheritance is to merge the data-holding behaviour of the Event class with the logic of how the event has to be processed. This is particularly useful in simplifying the process of writing the user analysis code since it presents the user with a common and familiar interface to the data.

Before starting to process the data the plugin manager loads all the user plugins and replaces the user plugin data pointers with its own. The data processing is then performed 
according to the usual steps in a TSelector analysis, with the only caveat that in each of the classic TSelector methods the TSAnaPluginManager takes care of calling the same method for each of the user plugins.

Listing 1. The plugin manager schedules execution of the user plugins

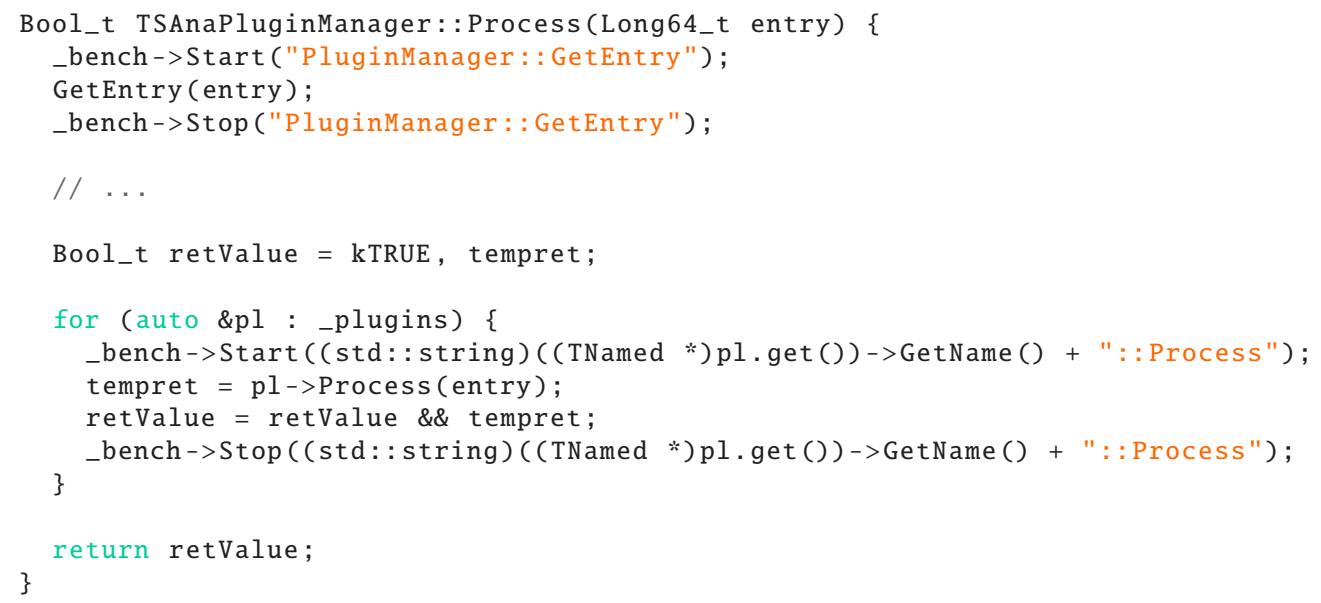

A set of custom stopwatches measures the execution time of each user plugin as well as the time required to load the data in memory, to profile and possibly spot bottlenecks in the user code.

The resulting execution flow is summarised in Figure 2: the purple blocks show all the tasks that are handled by the plugin manager, and thus only executed once per event, while specialization is delegated to the user plugins only where needed.

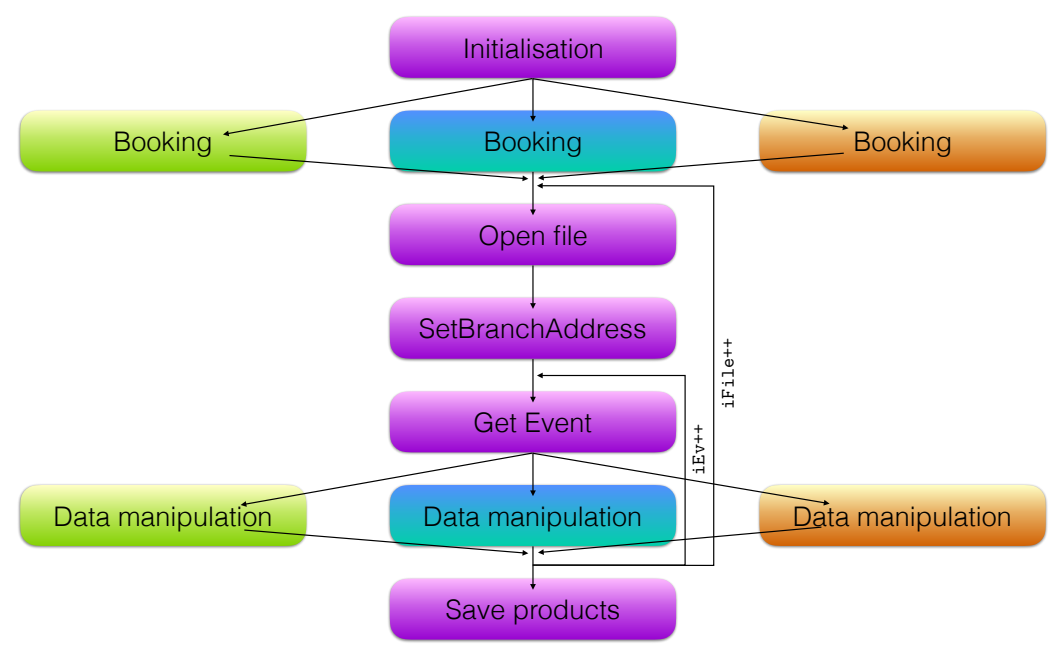

Figure 2. Execution flow of the analysis process. All common operation are handled by the plugin manager (purple blocks), whereas the users plugins introduce the required specialisation (green, blue, and orange blocks). 


\subsection{The user plugins}

The class TSAnaPlugin defines a base interface for user plugins. It inherits from both the TSelector and the custom Event class, same as for the plugin manager, and it will be the one from which all user plugins shall inherit.

The TSAnaPlugin class implements all the TSelector members and it exposes to the user an additional hook where the user can book the desired output objects (see Sec. 2.3). The two main methods where the user will write his analysis code are:

\section{- TSAnaPlugin: : BookHistos()}

This member function is called by the plugin manager as soon as the user plugin is first created, in the initialization phase. Here the user can declare all the analysis objects that must be created or initialized before the event loop. Special care has been given to the creation of ROOT histograms and trees, which is handled by two dedicated managers (described in Sec. 2.3).

\section{- TSAnaPlugin: :Process()}

This member function is called by the plugin manager each time a new event is loaded in memory. It contains the main body of the analysis, such as event selections, variables manipulation, histogram/tree filling and so on. As a design choice, the fact that the TSAnaPlugin class inherits from the analysis custom Event class means that in this method all the pointers to the internal data structures are already available to the user for utilization without the need to redefine/redeclare them. The user can, then, simply write the same kind of analysis code he would have written in his custom programs or macros.

Listing 2. Example user analysis code

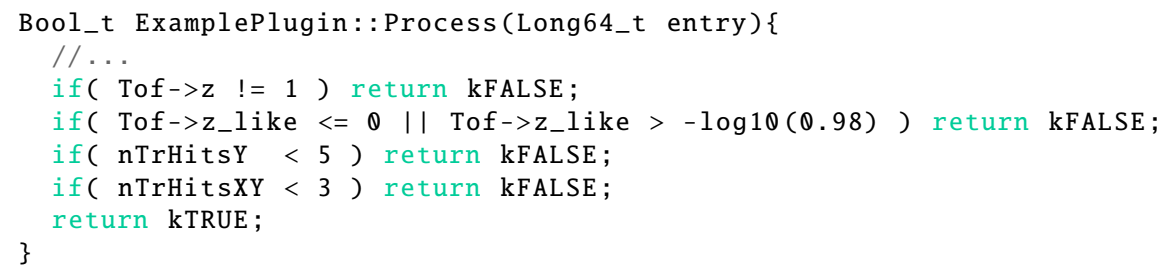

One small detail worth mentioning is that the Process () method needs to return a Bool_t even if this return value is not used in the TSelector scheme, we chose to follow the original implementation by the ROOT team. This means that if a user wants to skip processing an event he should just return kFALSE.

Inheriting from TSelector means that in principle a user plugin can be run in "standalone mode", i.e. without a plugin manager. The framework allows for this possibility: if a plugin manager is not present then the user plugin will take care of the data loading, otherwise the plugin manager will override all the pointers to the internal data structures in the TSAnaPlugin object with its own (in this way no data is actually moved, and it is still owned by the plugin manager). Standalone execution is especially useful if the user wants to test his own code before scheduling it for execution.

\subsection{Output handling}

The most common analysis workflow in HEP is looping on a given set of events, discard those not considered interesting for the analysis, and then either filling a set of histograms with the distribution of some relevant observables or save a golden subset of events in a ROOT TTree for a more detailed analysis. 
For this reason the framework provides an interface to handle histogram and tree creation and manipulation in a quick and transparent way. The plugin manager holds two objects: a histogram manager and a tree manager. The user plugins will then forward all the requested operations on histogram/trees to the plugin manager.

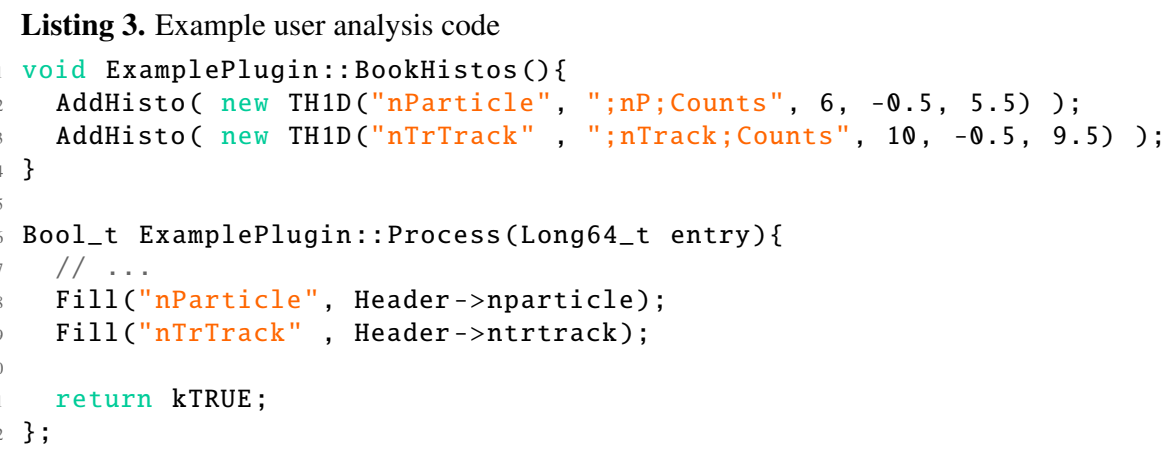

Histogram and trees are addressed by name and, to avoid collision, the plugin manager combines the histogram/tree name with the user plugin name in order to create a unique name for each objects it needs to store and access. At the end of the event loop the objects created by each plugin are saved in a dedicated TDirectory inside the output TFile. This is done to ease the file management in batch jobs, in this way there is no need to know how many plugins have been run and how many files were created: the output file is always one and the compartmentalization happens inside the TFile itself.

\subsection{The selection store}

Data loading is the most time-consuming task in the analysis that should not be replicated by all the users, but it is not the only one. Event selection is typically another task that is performed in the same way by different users and that could benefit from a shared approach.

It is very likely that two or more users share part of the selections they use to perform their analysis, especially the more basic ones that are usually related to ensure a good reconstruction quality for the events under study.

The framework offers to the users an infrastructure that allows defining and creating selections in such a way that they will be

1. Possibly visible and usable by all the other users.

2. Automatically cached.

This is implemented by a chained class structure where the most basic entity is the CutAction, which is basically a named, parametrised lambda function that accepts an Event object and return a bool expressing wether the event passes the selection or not.

Listing 4. The CutAction class

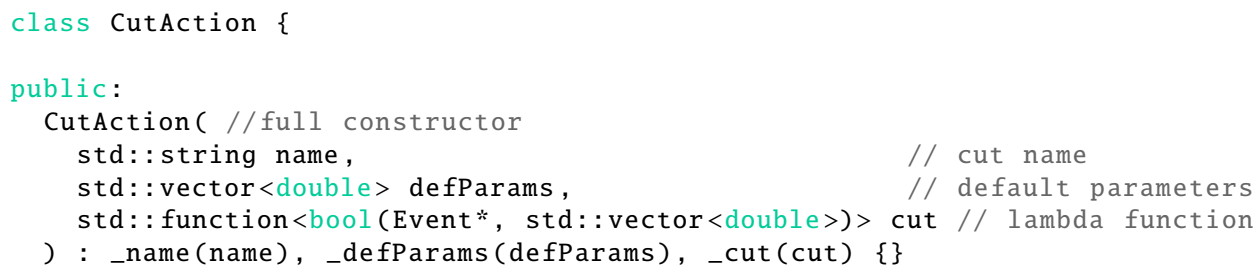




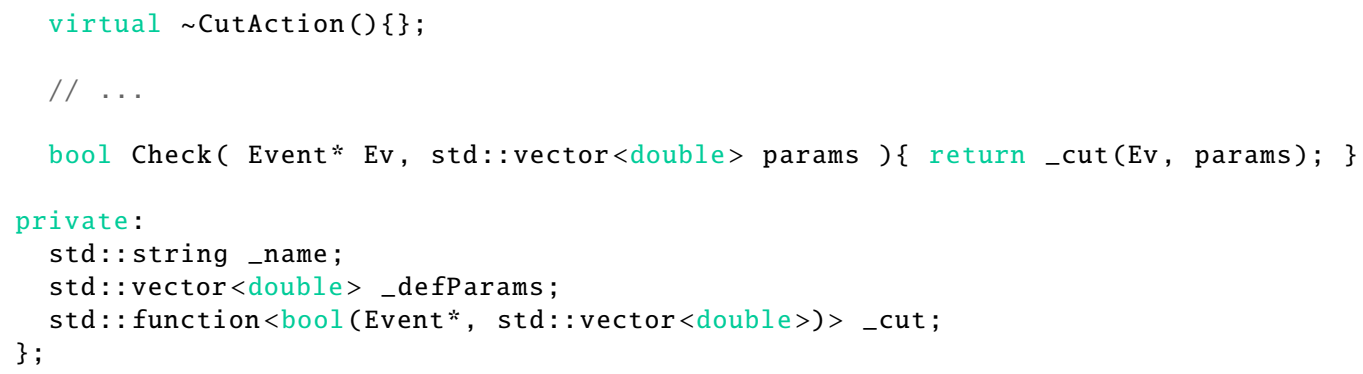

The most common and used selections have already been implemented but if the user needs to define a very specific selection he can easily write his own CutAction.

Listing 5. An example CutAction which checks is the event contains exactly $n$ tracks. The default value for $n$ is 1 .

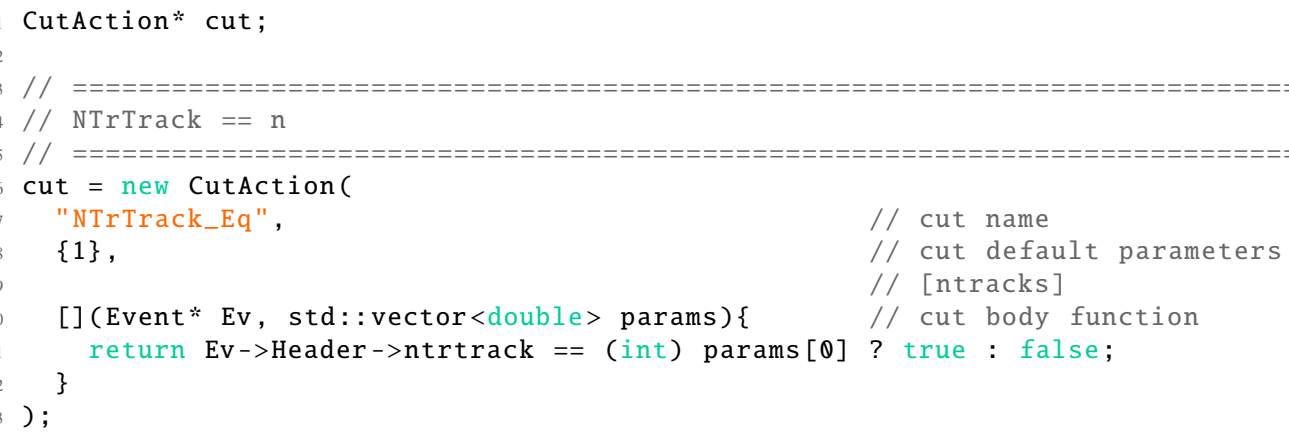

CutAction objects are not directly exposed to the user during the event loop, but rather they are hidden behind a CutProxy object that handles the chaching of the CutAction result for the same combination of event and parameters.

The realisation of a specific CutAction with a given set of parameters defines a specific CutInstance. CutInstance objects are the ones available to the user during the event loop and there might be one or more CutInstance sharing the same CutProxy but evaluated with different parameters.

CutInstances can be chained in CutList objects. These lists and instances are held in CutMgr objects that handle the requests by the users during the event loop. Each user plugin holds a CutMgr that stores the user-defined selections. If at runtime the user requests a list or selection to be evaluated which is not present in the plugin CutMgr, then it will automatically fall back to the CutMgr object in the plugin manager. If the selection is missing from the plugin manager CutMgr as well, the final fallback is made on a precompiled list of selections, called "selection store", held in a global CutDB object. The idea behind this logic is that the most common selections should be available to everyone in the "selection store" while selections needed only by one or few plugins should be put in the plugin manager CutMgr. On top of this each user can actually reimplement a given selection in a custom way by creating and adding the corresponding CutAction and CutInstance to the plugin CutMgr. This fallback mechanic allows a user to replicate an entire analysis flow adding the ability to replace or redefine a subset of selections without modifying everything.

The final scheme for the selection logic handling is depicted in Figure 3. 

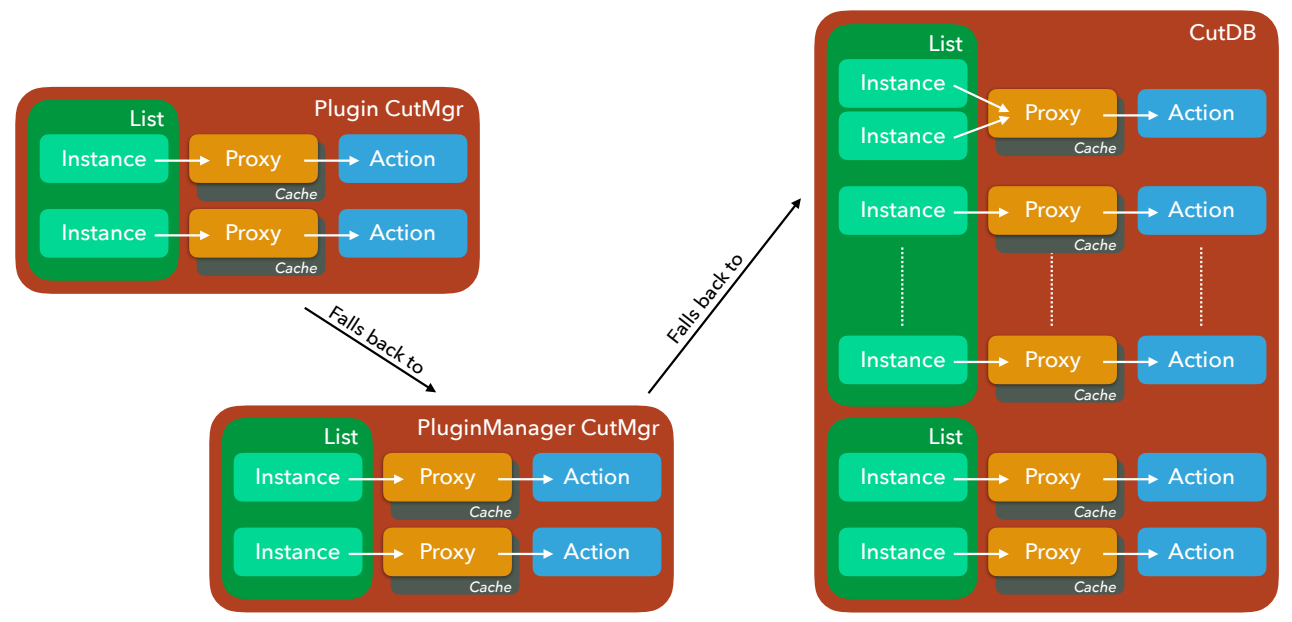

Figure 3. Logic diagram for accessing selections from a user plugin.

\section{Conclusions}

A framework is being developed within the AMS-Alpha dbar analysis group to encapsulate code from each user and share all the I/O operations. The framework leverages the TSelector structure to encapsulate code into dynamically linked libraries that can be loaded at runtime by a plugin manager that loads the data and runs each plugin on said data.

Users can easily define and fill histograms and trees which are then managed and saved by the plugin manager. Furthermore users can apply parametric selections to each event, and for each unique selection the result is calculated only once and then cached for following calls.

This work should allow the setup of nightly analysis trains to allow for day-scale iteration on the analysis and optimize user time.

Future improvements and addition to this framework are being planned and might include automatic performance checks (per-plugin cpu efficiency and memory usage), a webdashboard to monitor the status of the analysis train and much more.

\section{References}

[1] S. Ting, Nucl. Phys. B, Proc. Suppl. 243-244, 12 (2013)

[2] ROOT project, "Root" [software], version 6.14.04, 2018. Available from https://root. cern.ch/content/release-61404 